T. KOBAYASHI

KODAI MATH. J.

3 (1980), 8-25

\title{
PERMUTABILITY AND UNIQUE FACTORIZABILITY OF CERTAIN ENTIRE FUNCTIONS
}

\author{
By TADASHI KOBAYASH
}

Let $f(z)$ and $g(z)$ be entire functions. We say that $f(z)$ and $g(z)$ are permutable if they satisfy the relation

$$
f(g(z))=g(f(z))
$$

for every complex number $z$. Recently by several authers the permutability of entire functions have been discussed and various entire functions whose permutable functions can be determined have been listed up. However, as far as the present auther knows, there are a few methods for attacking this subject.

The purpose of this paper is to indicate a method by which permutable functions of some certain entire functions can be determined. The functions which we shall consider below are of a rather different kind from those previously investigated. Our method is very elementary in principle.

Let $f(z)$ be $z+e^{z}$, and let $g(z)$ be $z e^{z}$. Then Urabe [6] has shown the unique factorizability of the function $g(f(z))$. Naturally we are led to the problem of whether the function $f(g(z))$ is uniquely factorizable. In the final section, as an application of our technique, we shall prove the unique factorizability of this entire function.

In what follows, we freely use the symbols and the fundamental concepts of Nevanlinna theory.

1. In the factorization theory, it is well known that the entire function $z+e^{z}$ is prime. Firstly we shall decide all the entire functions of finite order which are permutable with $z+e^{z}$.

THEOREM 1. Let $f(z)$ be $z+C e^{a z}$, where $a$ and $C$ are constants with $a C \neq 0$. Let $g(z)$ be a nonconstant entire function of finite order which is permutable with $f(z)$. Then either $g(z)=f(z)+C_{*}$, or $g(z)=z+C_{*}$, where $C_{*}$ is a constant with $\exp \left(a C_{*}\right)=1$.

Evidently $f(z)=z+C e^{a z}$ satisfies the following differential equation

$$
f^{\prime}(z)-1=a(f(z)-z)=a C e^{a z} .
$$

Received October 16, 1978 
Further let us set $F(z)=f(f(z))$. Then for an arbitrary finite complex number $w$, the order of $\bar{N}(r, w, F)$ is infinite. This fact is easily verified by the second main theorem of Nevanlinna theory.

Now let $g(z)$ be a nonconstant entire function of finite order satisfying

$$
f(g(z))=g(f(z)) \text {. }
$$

Then we have

$$
f^{\prime}(g(z)) g^{\prime}(z)=g^{\prime}(f(z)) f^{\prime}(z) .
$$

Assume that $g^{\prime}(z)$ has no zeros. Then it follows from (1.3) that

$$
\bar{N}\left(r, w_{1}, g\right)+\bar{N}\left(r, w_{2}, g\right) \leqq N\left(r, 0, f^{\prime}\right)
$$

for values of $r$, where $w_{1}$ and $w_{2}$ are two distinct zero-points of $f^{\prime}(z)$. Hence $g(z)$ has at most order one, so that

$$
g^{\prime}(z)=\exp (A z+B)
$$

with suitable constants $A$ and $B$. If $A \neq 0$, then $g(z)$ has one finite lacunary value. This is clearly untenable. Therefore $g^{\prime}(z)$ must be constant, so that $g(z)$ is a linear function. Thus we have the desired result from the relation (1.2).

Hereafter we may assume that $g^{\prime}(z)$ has at least one zero-point. Let $s$ be a zero-point of $g^{\prime}(z)$. Then from (1.3), all the roots of $f(z)=s$ satisfy

$$
f^{\prime}(g(z)) g^{\prime}(z)=g^{\prime}(s) f^{\prime}(z)=0 .
$$

Firstly assume that all the roots of $f(z)=s$ satisfy $g^{\prime}(z)=0$. Let $t$ be a root of $F(z)=s$. Since $f(f(t))=s, g^{\prime}(f(t))=0$. Thus from $(1.3), g^{\prime}(t)=0$ or $f^{\prime}(g(t))=0$. If $f^{\prime}(g(t))=0$, then from the relation (1.1),

$$
g(f(t))-g(t)=C \exp (a g(t))=-\frac{1}{a} .
$$

Hence

$$
\begin{aligned}
g(s) & =f(g(f(t))) \\
& =g(f(t))+C \exp (a g(f(t))) \\
& =g(t)-\frac{1+e}{e a} .
\end{aligned}
$$

Consequently all the roots of $F(z)=s$ must satisfy either $g^{\prime}(z)=0$, or

$$
g(z)=g(s)+\frac{1+e}{e a}=A .
$$

Thus we have

$$
\bar{N}(r, s, F) \leqq N\left(r, 0, g^{\prime}\right)+N(r, A, g)
$$


for values or $r$. However, since the order of $g(z)$ is finite and that of $\bar{N}(r, s, F)$ is infinite, it is a contradiction.

Secondly we assume that there are two different roots $u$ and $v$ of $f(z)=s$ such that $g^{\prime}(u)=0$ and $g^{\prime}(v) \neq 0$. Then $f^{\prime}(g(v))=0$ by (1.4). It thus follows from (1.1) that

$$
a(g(s)-g(v))=a C \exp (a g(v))=-1 .
$$

Here we further assume that there is a root $w$ of $f(z)=u$ such that $g^{\prime}(w) \neq 0$. Then since $f^{\prime}(g(w))=0$,

$$
a(g(u)-g(w))=a C \exp (a g(w))=-1 .
$$

Hence we obtain

$$
g(s)=g(w)-\frac{1+e}{e a} .
$$

It therefore follows from these (1.5) and (1.6) that

and

$$
e a(g(w)-g(v))=1
$$

$$
\exp (a g(w)-a g(v))=1 \text {. }
$$

This is clearly impossible. Accordingly all the roots of $f(z)=u$ must be zeros of $g^{\prime}(z)$. However this is also untenable as shown just above. Consequently if $s$ is a zero-point of $g^{\prime}(z)$, then $g^{\prime}(z) \neq 0$ at every root of $f(z)=s$.

By this fact, we can claim that $g^{\prime}(f(z)) \neq 0$ at every zero-point of $g^{\prime}(z)$. In fact, if there is a zero-point $s$ of $g^{\prime}(z)$ such that $g^{\prime}(f(s))=0$, then by what mentioned just above, $g^{\prime}(z) \neq 0$ at every root of $f(z)=f(s)$. In particular, $g^{\prime}(s) \neq 0$. This is impossible. Furthermore from (1.3), we can conclude that all the zeros of $g^{\prime}(z)$ are also zeros of $f^{\prime}(z)$.

Let $s$ be a zero-point of $g^{\prime}(z)$ again. Then by the above fact and by (1.4), $f^{\prime}(g(z))=0$ at every root of $f(z)=s$. Hence from the equation

$$
\begin{aligned}
f^{\prime \prime}(g(z)) & \left(g^{\prime}(z)\right)^{2}+f^{\prime}(g(z)) g^{\prime \prime}(z) \\
= & g^{\prime \prime}(f(z))\left(f^{\prime}(z)\right)^{2}+g^{\prime}(f(z)) f^{\prime \prime}(z),
\end{aligned}
$$

all the roots of $f(z)=s$ satisfy

$$
f^{\prime \prime}(g(z))\left(g^{\prime}(z)\right)^{2}=g^{\prime \prime}(s)\left(f^{\prime}(z)\right)^{2} .
$$

Here let us notice that $a f^{\prime}(z)=a+f^{\prime \prime}(z)$ for each complex number $z$. Then $a+f^{\prime \prime}(g(z))=0$, so that by $(1.7)$,

$$
a\left(g^{\prime}(z)\right)^{2}+g^{\prime \prime}(s)\left(f^{\prime}(z)\right)^{2}=0
$$

at every root of $f(z)=s$. Further we have $g^{\prime \prime}(s) \neq 0$.

Now let $t$ be a root of $F(z)=s$. Then 


$$
g^{\prime}(f(t)) f^{\prime}(t)=f^{\prime}(g(t)) g^{\prime}(t) .
$$

Since $f(f(t))=s$, the above (1.8) implies

$$
a\left(g^{\prime}(f(t))\right)^{2}+g^{\prime \prime}(s)\left(f^{\prime}(f(t))\right)^{2}=0 .
$$

Furthermore by (1.1),

$$
\begin{aligned}
& f^{\prime}(f(t))=1+a s-a f(t), \\
& f^{\prime}(g(t))=1+a g(f(t))-a g(t),
\end{aligned}
$$

and

$$
\begin{aligned}
f^{\prime}(g(f(t))) & =1+a(f(g(f(t)))-g(f(t))) \\
& =1+a g(s)-a g(f(t)) .
\end{aligned}
$$

Since $f(f(t))=s, f^{\prime}(g(f(t)))=0$. We therefore have

$$
1+a g(s)-a g(f(t))=0,
$$

so that

$$
f^{\prime}(g(t))=2+a g(s)-a g(t) .
$$

Substituting these (1.9), (1.11) and (1.12) into (1.10), we finally obtain

$$
\begin{aligned}
-a\left(g^{\prime}(t)\right)^{2} & (2+a g(s)-a g(t))^{2} \\
= & g^{\prime \prime}(s)\left(f^{\prime}(t)\right)^{2}(1+a s-a f(t))^{2} .
\end{aligned}
$$

This (1.13) means that all the roots of $F(z)=s$ must be zeros of the entire function $G(z)$ defined by

Therefore

$$
\begin{aligned}
G(z)= & a\left(g^{\prime}(z)\right)^{2}(2+a g(s)-a g(z))^{2} \\
& +g^{\prime \prime}(s)\left(f^{\prime}(z)\right)^{2}(1+a s-a f(z))^{2} .
\end{aligned}
$$

$$
\bar{N}(r, s, F) \leqq N(r, 0, G)
$$

for values of $r$. On the other hand it is clear that the order of $G(z)$ is finite. Hereby the function $G(z)$ must be identically equal to zero. Accordingly with a suitable non-zero constant $A$, we have

$$
\begin{aligned}
A f^{\prime}(z)(1 & +a s-a f(z)) \\
& =g^{\prime}(z)(2+a g(s)-a g(z))
\end{aligned}
$$

for each complex number $z$. Further from this (1.14), we can assert that

$$
\begin{aligned}
a A(f(z))^{2} & -2(1+a s) A f(z)+B \\
= & a(g(z))^{2}-2(2+a g(s)) g(z),
\end{aligned}
$$


where $B$ is a certain constant. Here we must determine this constant $B$. To do so, let $x$ be a root of $a f(z)=1+a s$. Then $f^{\prime}(x)=2+a s-a x$ by (1.1). Hence we may assume that $f^{\prime}(x) \neq 0$. Thus $g^{\prime}(x) \neq 0$ and $a g(x)=2+a g(s)$ by (1.14). Therefore the equation (1.15) implies

$$
a B+(2+a g(s))^{2}=A(1+a s)^{2},
$$

so that

$$
(a g(z)-2-a g(s))^{2}=A(a f(z)-1-a s)^{2}
$$

for every complex number $z$. Consequently $g(z)$ can be written in the form

$$
g(z)=A^{*} f(z)+B^{*},
$$

where $A^{*}$ and $B^{*}$ are constants with $A^{*} \neq 0$. Here taking into account of (1.2), we obtain the desired result immediately. This completes the proof of Theorem 1.

2. We shall consider one more function whose permutable functions can be determined by our method.

Let $f(z)$ be $z+\sin (z+c)$ with a constant $c$. Then $f(z)$ satisfies the following differential equations

$$
\begin{aligned}
& \left(f^{\prime}(z)-1\right)^{2}+(f(z)-z)^{2}=1, \\
& f^{\prime \prime}(z)+f(z)=z .
\end{aligned}
$$

Hereafter let us denote the function $f(f(z))$ by $F(z)$. Then for every finite complex number $w, \bar{N}(r, w, F)$ is of infinite order.

Now let $g(z)$ be a nonconstant entire function of finite order which is permutable with $f(z)$. Then

$$
f(g(z))=g(f(z))
$$

so that

$$
f^{\prime}(g(z)) g^{\prime}(z)=g^{\prime}(f(z)) f^{\prime}(z)
$$

for every complex number $z$. For a moment assume that $g^{\prime}(z)$ has no zeros. Then $g(z)$ must be linear by the same reason as in the proof of Theorem 1 . Since $g(z)$ satisfies (2.2), we can conclude that either $g(z)=z+C^{*}$ with a constant $C^{*}$ satisfying $\cos C^{*}=1$, or $g(z)=C_{*}-z$ with a constant $C_{*}$ such as $\cos \left(C_{*}+2 c\right)=1$.

In what follows we therefore assume that $g^{\prime}(z)$ has at least one zero-point. Let $s$ be a zero-point of $g^{\prime}(z)$. Then by (2.3),

$$
f^{\prime}(g(z)) g^{\prime}(z)=g^{\prime}(s) f^{\prime}(z)=0
$$

at every root of $f(z)=s$. Here assume that there is a root $t$ of $f(z)=s$ such 
that $f^{\prime}(g(t)) \neq 0$. Then $g^{\prime}(t)=0$ and all the roots of $f(z)=t$ satisfy

$$
f^{\prime}(g(z)) g^{\prime}(z)=g^{\prime}(t) f^{\prime}(z)=0 .
$$

Hence if there is a root $u$ of $f(z)=t$ such that $g^{\prime}(u) \neq 0$, then $f^{\prime}(g(u))=0$, so that $g(u)=f(g(u))=g(t)$ by (2.1). This implies $f^{\prime}(g(t))=0$, which is absurd. Therefore all the roots of $f(z)=t$ are zeros of $g^{\prime}(z)$. Further let $w$ be a root of $F(z)=t$. Then $g^{\prime}(f(w))=0$, so that $f^{\prime}(g(w))=0$ or $g^{\prime}(w)=0$ by (2.3). If $f^{\prime}(g(w))=0$, then $f(g(w))=g(w)$, so $g(w)=g(t)$. This is absurd again. By these facts, all the roots of $F(z)=t$ must be zeros of $g^{\prime}(z)$. Since the order of $g(z)$ is finite and $\bar{N}(r, t, F)$ is of infinite order, this is clearly impossible. Consequently all the roots of $f(z)=s$ satisfy $f^{\prime}(g(z))=0$. Hence by $(2.1), g(z)=g(s)$ at these roots.

Again let $s$ be a zero-point of $g^{\prime}(z)$. Then by what mentioned just above, $f^{\prime}(g(z))=0$ and $g(z)=g(s)$ at every root of $f(z)=s$. Therefore from (2.1), all the roots of $f(z)=s$ satisfy

$$
f^{\prime \prime}(g(z))=0, \quad f^{(3)}(g(z))=1 .
$$

Further it follows from (2.3) that

so that

$$
g^{\prime \prime}(s)\left(f^{\prime}(z)\right)^{2}+g^{\prime}(s) f^{\prime \prime}(z)=f^{\prime \prime}(g(z))\left(g^{\prime}(z)\right)^{2}+f^{\prime}(g(z)) g^{\prime \prime}(z)=0,
$$

$$
g^{\prime \prime}(s)\left(f^{\prime}(z)\right)^{2}=0
$$

at these roots. Evidently by (2.1), with one possible exception, all the roots of $f(z)=s$ are simple. Hence the above (2.4) implies $g^{\prime \prime}(s)=0$. Accordingly by these facts, we obtain

$$
\left(g^{\prime}(z)\right)^{3}=g^{(3)}(s)\left(f^{\prime}(z)\right)^{3}
$$

at every root of $f(z)=s$. Here we can claim that $g^{(3)}(s) \neq 0$. Indeed if $g^{(3)}(s)=0$, all the roots of $f(z)=s$ are zeros of $g^{\prime}(z)$. Thus for each root $u$ of $F(z)=s$, $g^{\prime}(f(u))=0$, so that either $f^{\prime}(g(u))=0$ or $g^{\prime}(u)=0$. If $f^{\prime}(g(u))=0$, then $(2.1)$ yields $g(u)=g(f(u))=g(s)$. Hence we have

$$
\bar{N}(r, s, F) \leqq N\left(r, 0, g^{\prime}\right)+N(r, g(s), g)
$$

for values of $r$. This is clearly absurd. Hereby $g^{(3)}(s) \neq 0$ as we claim.

Now let $t$ be a root of $F(z)=s$. Then the above (2.5) implies

$$
\left(g^{\prime}(f(t))\right)^{3}=g^{(3)}(s)\left(f^{\prime}(f(t))\right)^{3} .
$$

Further since $f(g(t))=g(f(t))=g(s),(2.1)$ and (2.3) yield

$$
\begin{aligned}
& f^{\prime}(g(t)) g^{\prime}(t)=g^{\prime}(f(t)) f^{\prime}(t), \\
& \left(f^{\prime}(f(t))-1\right)^{2}+(s-f(t))^{2}=1, \\
& \left(f^{\prime}(g(t))-1\right)^{2}+(g(s)-g(t))^{2}=1 .
\end{aligned}
$$


Here by $A_{1}, A_{2}$ and $A_{3}$, we denote the three roots of $z^{3}=g^{(3)}(s)$. With these $A_{n}$, let us introduce the following entire functions $G_{n}(z)$ defined by

$$
\begin{aligned}
G_{n}(z)= & A_{n}{ }^{2}\left(f^{\prime}(z)\right)^{2}(s-f(z))^{2} \\
& -\left(g^{\prime}(z)\right)^{2}(g(z)-g(s))^{2} .
\end{aligned}
$$

Further let us set

$$
\begin{aligned}
S_{n}(z)= & \left(G_{n}(z)\right)^{2}-4 A_{n} f^{\prime}(z)\left(A_{n} f^{\prime}(z)-g^{\prime}(z)\right) G_{n}(z) \\
& +4 A_{n}{ }^{2}(s-f(z))^{2}\left(f^{\prime}(z)\right)^{2}\left(A_{n} f^{\prime}(z)-g^{\prime}(z)\right)^{2}, \\
S(z)= & S_{1}(z) S_{2}(z) S_{3}(z) .
\end{aligned}
$$

Obviously these entire functions $G_{n}(z), S_{n}(z)$ and $S(z)$ are of finite order.

We are now in a position to obtain our desired result at hand. Substituting (2.7) into (2.6), and by making use of the above functions, after a simple calculation, we can finally see that $S(t)=0$. This means that all the roots of $F(z)=s$ must be zeros of $S(z)$, so that

$$
\bar{N}(r, s, F) \leqq N(r, 0, S)
$$

for values of $r$. Since the order of $S(z)$ is finite and that of $\bar{N}(r, s, F)$ is infinite, the entire function $S(z)$ must be identically equal to zero. Therefore at least one of the three functions $S_{n}(z)$ vanishes identically. Consequently we find

$$
\begin{aligned}
(G(z))^{2}- & 4 A f^{\prime}(z)\left(A f^{\prime}(z)-g^{\prime}(z)\right) G(z) \\
& +4 A^{2}\left(f^{\prime}(z)\right)^{2}(s-f(z))^{2}\left(A f^{\prime}(z)-g^{\prime}(z)\right)^{2}=0
\end{aligned}
$$

for each complex number $z$, where

$$
\begin{aligned}
G(z)= & A^{2}\left(f^{\prime}(z)\right)^{2}(s-f(z))^{2} \\
& -\left(g^{\prime}(z)\right)^{2}(g(z)-g(s))^{2},
\end{aligned}
$$

and $A$ is a constant with $A^{3}=g^{(3)}(s) \neq 0$. Let us set

$$
L(z)=2 A f^{\prime}(z)\left(A f^{\prime}(z)-g^{\prime}(z)\right) .
$$

Then the above (2.8) can be rewritten as

$$
(G(z)-L(z))^{2}=(L(z))^{2}\left(1-(s-f(z))^{2}\right) .
$$

We now assume that $L(z)$ is not identically zero. Then

$$
H(z)=\frac{G(z)-L(z)}{L(z)}
$$

is an entire function and satisfies

$$
(H(z))^{2}=1-(s-f(z))^{2}
$$


for every complex number $z$. On the other hand it is possible to find a number $x$ such that $f(x)=s-1$ and $f^{\prime}(x) \neq 0$. Clearly $H(x)=0$ and

$$
2(s-f(x)) f^{\prime}(x)=2 H(x) H^{\prime}(x)=0,
$$

so that $2 f^{\prime}(x)=0$. This is a contradiction. Therefore $L(z)$ must be identically equal to zero. Hereby

$$
g^{\prime}(z)=A f^{\prime}(z)
$$

for every complex number $z$. Consequently $g(z)$ can be written in the form

$$
g(z)=A f(z)+B,
$$

where $A$ and $B$ are constants with $A \neq 0$. By taking into account of (2.2), we now obtain the following theorem.

THEOREM 2. Let $f(z)$ be $z+\sin (z+c)$ with a constant c. Let $g(z)$ be a transcendental entire function of finite order which is permutable with $f(z)$. Then either $g(z)=f(z)+c^{*}$ with a constant $c^{*}$ satısfying $\cos c^{*}=1$, or $g(z)=c_{*}-f(z)$ with a constant such as $\cos \left(c_{*}+2 c\right)=1$.

3. In this final section we shall present an example on unique factorizability.

THEOREM 3. Let $f(z)$ be $z+e^{z}$, and let $g(z)$ be $z e^{z}$. Then the entire function $f(g(z))$ is uniquely factorizable.

The proof of this fact depends on our principle. Before proceeding with the proof, we sum up some properties of these functions $f(z)$ and $g(z)$ which we require in the sequel.

Let us set $H(z)=f(g(z))$. Then $H(z)$ satisfies

$$
\begin{aligned}
H^{\prime}(z) & =f^{\prime}(g(z)) g^{\prime}(z) \\
& =\left(1+\exp \left(z e^{z}\right)\right)(1+z) e^{z} \\
& =\left(1-z e^{z}+H(z)\right)(1+z) e^{z} .
\end{aligned}
$$

In particular, $H(0)=1$ and $H^{\prime}(0)=2$. Furthermore all the zeros of $H^{\prime}(z)$ are simple.

Let $c$ be a real positive number with $1+\log c>0$. Now we consider the level line $L(c)=\{z:|g(z)|=c\}$. Then by an elementary calculation, we can assert that $L(c)$ is a single analytic curve in the whole finite plane and is symmetric with respect to the real axis. Furthermore $L(c)$ is contained entirely in the closed domain

$$
\{z: \operatorname{Re} z \leqq c,|\arg z| \leqq \theta(c)\},
$$

where $\theta(c)$ is the root of $\cos x=-1 / e c$ with $0<x<\pi$. 
Finally let us notice that

$$
\lim _{r \rightarrow \infty} \frac{N(r, w, g)}{r}=\frac{1}{\pi}
$$

for every finite complex number $w$ other than $w=0$. This fact is easily verified by the second main theorem.

Proof. Let $F(z)$ and $G(z)$ be nonconstant entire functions satisfying

$$
H(z)=f(g(z))=F(G(z)) .
$$

Then

$$
H^{\prime}(z)=f^{\prime}(g(z)) g^{\prime}(z)=F^{\prime}(G(z)) G^{\prime}(z) .
$$

Since $H(0)=1$ and $H^{\prime}(0)=2$, we may assume that $G(0)=0, F(0)=1, G^{\prime}(0)=1$ and $F^{\prime}(0)=2$. Our goal is to show that (1) $G(z)=z$ and $F(z)=H(z)$, (2) $F(z)=2 z+1$ and $2 G(z)=H(z)-1$, or (3) $G(z)=g(z)$ and $F(z)=f(z)$. Hereafter our consideration is divided into several steps, since it needs a little bit complicated process.

The first step. Assume that $F^{\prime}(z)$ has no zeros. It then follows from (3.1) and (3.3) that

$$
N\left(r, 0, G^{\prime}(z)\right)=N(r,-1, \exp (g(z)))+\log ^{+} r
$$

for values of $r$. Here remark that

$$
\lim _{r \rightarrow \infty} \frac{N(r, w, \exp (g(z)))}{T(r, \exp (g(z)))}=1
$$

for every finite complex number $w$ other than $w=0$. This less trivial fact can be verified by making use of the second main tneorem. Therefore (3.4) and (3.5) imply

$$
\lim _{r \rightarrow \infty} \frac{N\left(r, 0, G^{\prime}(z)\right)}{T(r, H(z))}=1
$$

so that

$$
\liminf _{r \rightarrow \infty} \frac{T\left(r, G^{\prime}\right)}{T(r, H)} \geqq 1 .
$$

On the other hand if $F(z)$ is transcendental, then $T(r, G)=o(T(r, H))$, so that

$$
\liminf _{r \rightarrow \infty} \frac{T\left(r, G^{\prime}\right)}{T(r, H)}=0 \text {. }
$$

This clearly contradicts (3.6). Hence $F(z)$ reduces to a polynomial. Accordingly, $F(z)=2 z+1$, so $G(z)=(H(z)-1) / 2$.

The second step. In this step, let us assume that $F^{\prime}(z)$ has only one zeropoint $s$. Then 


$$
\begin{aligned}
& N(r, s, G(z))+N\left(r, 0, G^{\prime}(z)\right) \\
& \quad=N(r,-1, \exp (g(z)))+\log ^{+} r
\end{aligned}
$$

for values of $r$. Hence by the same reason as in the above step,

$$
\lim _{r \rightarrow \infty} \frac{N(r, s, G)+N\left(r, 0, G^{\prime}\right)}{T(r, H)}=1,
$$

so that $F(z)$ must be a polynomial. Therefore $F(z)$ can be written in the form

$$
F(z)=A(z-s)^{n}+B,
$$

where $A$ and $B$ are constants with $A \neq 0$, and $n$ is an integer with $n \geqq 2$. It thus follows that

$$
\begin{aligned}
H(z) & =A(G(z)-s)^{n}+B=g(z)+\exp (g(z)), \\
H^{\prime}(z) & =n A(G(z)-s)^{n-1} G^{\prime}(z) \\
& =(1+\exp (g(z)))(1+z) e^{z} .
\end{aligned}
$$

Let $t$ be a root of $G(z)=s$. Then by (3.8), either $t=-1$ or else $\mathrm{i}_{\mathrm{i}} g(t)=B+1$. This means that

$$
N(r, s, G) \leqq N(r, 1+B, g)+\log ^{+} r,
$$

so that $N(r, s, G)=O(r)$. Hereby from (3.7), we have

$$
\lim _{r \rightarrow \infty} \frac{N\left(r, 0, G^{\prime}\right)}{T(r, H)}=1 \text {. }
$$

Furthermore we can express the function $G(z)$ as

$$
G(z)=s+L(z) \exp (K(z)),
$$

where $K(z)$ is entire and $L(z)$ is the canonical product formed ${ }_{\lrcorner}^{*}$ by the zeros of $G(z)-s$. Since

$$
G^{\prime}(z)=\left(L^{\prime}(z)+L(z) K^{\prime}(z)\right) \exp (K(z)),
$$

we thus find

$$
\begin{aligned}
N\left(r, 0, G^{\prime}\right) & =N\left(r, 0, L^{\prime}+L K^{\prime}\right) \\
& \leqq T\left(r, L^{\prime}\right)+T(r, L)+T\left(r, K^{\prime}\right)+O(1) \\
& \leqq o\left(r^{2}\right)+T\left(r, K^{\prime}\right) .
\end{aligned}
$$

Combining this inequality with (3.9), we obtain

$$
\limsup _{r \rightarrow \infty} \frac{T(r, K)}{T(r, H)} \geqq 1 .
$$

On the other hand, by virtue of (3.8) and (3.10), 


$$
\lim _{r \rightarrow \infty} \frac{T(r, \exp (K(z)))}{T(r, H(z))}=\frac{1}{n} .
$$

Hence $T(r, K)=o(T(r, H))$, which is absurd by (3.11).

The third step. Next we assume that $F^{\prime}(z)$ has finitely many zeros $w_{\jmath}(1 \leqq \jmath \leqq n)$. Here we may assume that $n \geqq 2$. From (3.1),

$$
\begin{aligned}
H^{\prime}(z) & =F^{\prime}(G(z)) G^{\prime}(z) \\
& =(1+\exp (g(z))) g^{\prime}(z) \\
& =(1-g(z)+F(G(z))) g^{\prime}(z) .
\end{aligned}
$$

Hence every root of $G(z)=w$, is simple and satisfies either $g^{\prime}(z)=0$ or else $g(z)=1+F\left(w_{j}\right)$. It thus follows that

$$
N\left(r, w_{\jmath}, G\right) \leqq N\left(r, 1+F\left(w_{\jmath}\right), g\right)+\log ^{+} r=O(r)
$$

for each $w_{j}(1 \leqq j \leqq n)$, so that the order of $G(z)$ is at most one. On the other hand the relation (3.12) also gives

$$
\begin{aligned}
N(r,-1, \exp (g(z))) & \leqq N\left(r, 0, G^{\prime}(z)\right) \\
& +\sum_{\jmath=1}^{n} N\left(r, w_{\jmath}, G(z)\right)
\end{aligned}
$$

for values of $r$. Since the right hand side is of finite order but the left hand side is of infinite order, this inequality is clearly impossible.

The fourth step. In what follows, by the results of the above three steps, we may assume that $F(z)$ is transcendental and that $F^{\prime}(z)$ has infinitely many zeros. As in the third step, for each zero-point $w$ of $F^{\prime}(z)$, all the roots of $G(z)=w$ satisfy $\exp (g(z))=-1$ and $g(z)=1+F(w)$ unless $z=-1$. Hence we have $T(r, G)=O(r)$.

Now assume that $G^{\prime}(-1) \neq 0$. Then we can assert that $G(z)$ takes the value $G(-1)$ only at the point $z=-1$. In fact, let $t$ be a root of $G(z)=G(-1)$. Evidently $H(t)=H(-1)$, so that $H(t)$ is real. Further since $G^{\prime}(-1) \neq 0, F^{\prime}(G(-1))$ $=0$. Hence $F^{\prime}(G(t))=0$ and $H^{\prime}(t)=0$. It thus follows from (3.12) that $\exp (g(t))$ $=-1$, so that $H(t)=g(t)-1$ unless $t=-1$. Therefore if $t \neq-1, H(t)$ is not real. This is absurd. Hereby $G(z)-G(-1)$ has no zeros other than $z=-1$, provided $G^{\prime}(-1) \neq 0$.

Let $c_{n}$ be $(2 n+1) \pi i$ for each integer $n$. By $E_{n}$, we denote the set defined by

$$
\left\{w: F^{\prime}(w)=0, F(w)=c_{n}-1\right\} .
$$

Here let us assume that for some integer $k$, the set $E_{k}$ consists of infinitely many points. Let $w_{1}, \cdots, w_{m}$ be arbitrary $m$ points of $E_{k}$. Then by virtue of the relation (3.12), all the roots of $G(z)=w$, satisfy $g(z)=c_{k}$ unless $z=-1$. Hence we have 


$$
\sum_{j=1}^{m} N\left(r, w_{\jmath}, G\right) \leqq N\left(r, c_{k}, g\right)+\log ^{+} r
$$

for values of $r$. Therefore the second main theorem implies

so that

$$
(m-1+o(1)) T(r, G) \leqq N\left(r, c_{k}, g\right),
$$

$$
\limsup _{r \rightarrow \infty} \frac{T(r, G)}{r} \leqq \frac{1}{(m-1) \pi} .
$$

Since the set $E_{k}$ is infinite, we can choose $m$ as large as we please. We thus have $T(r, G)=o(r)$. Furthermore it is clear that the set $E_{k}$ is unbounded and for each point $w$ of $E_{k}$, all the roots of $G(z)=w$ satisfy $g(z)=c_{k}$ unless $z=-1$. Hence these roots are distributed in the half plane $\operatorname{Re} z \leqq\left|c_{k}\right|$. By these facts, $G(z)$ must reduce to a polynomial of degree at most two [2]. If $G^{\prime}(-1)=0$, then we can write $G(z)$ as

$$
G(z)=A(z+1)^{2}+B
$$

with constants $A$ and $B$. Hence $G(0)=G(-2)$, so $H(0)=H(-2)$. This is absurd. Therefore $G^{\prime}(-1) \neq 0$. Hereby $G(z)$ takes the value $G(-1)$ only at the point $z=-1$. Consequently $G(z)$ is linear, so that $G(z)=z$.

The fifth step. Taking account of the result just above, we may assume that all the sets $E_{n}$ are finite sets. In this step we show some other properties of the function $G(z)$.

Firstly we shall prove that $G^{\prime}(-1)=0$ unless $G(z)=z$. To do so, let us assume that $G^{\prime}(-1) \neq 0$ again. Then $G(z)-G(-1)$ has no zeros other than $z=-1$. Since $T(r, G)=O(r)$, with suitable constants $a$ and $b$, we can write $G(z)$ as

$$
G(z)=G(-1)+(z+1) \exp (a z+b) .
$$

Here we may assume that $a \neq 0$. Evidently

$$
G^{\prime}(z)=(a z+a+1) \exp (a z+b) .
$$

Now we choose a number $c_{m}=(2 m+1) \pi \imath$ such as $H(u) \neq c_{m}-1$, where $u=-1-1 / a$. Then for every root $s$ of $g(z)=c_{m}, G(s)$ is a point of the set $E_{m}$ which is defined by (3.13). Actually if $g(s)=c_{m}$, then $H(s)=c_{m}-1$ and $H^{\prime}(s)=0$ by (3.12). Hence $F^{\prime}(G(s))=0$ since $s \neq u$. Clearly $G(s)$ is a point of $E_{m}$. Conversely let $w$ be a point of $E_{m}$ and let $t$ be a root of $G(z)=w$. Then $F^{\prime}(G(t))=0$ and $H(t)$ $=c_{m}-1$. Since $t \neq-1, H(t)=g(t)-1$, so that $g(t)=c_{m}$. Setting

$$
Q(z)=\prod_{j=1}^{N}\left(z-w_{j}\right),
$$

where $w_{1}, \cdots, w_{N}$ are all the points of the set $E_{m}$, we therefore see that the functions $Q(G(z))$ and $g(z)-c_{m}$ have the same zeros. Hence with suitable constants $a^{*}$ and $b^{*}$, 


$$
g(z)-c_{m}=Q(G(z)) \exp \left(a^{*} z+b^{*}\right) .
$$

Further let $s^{*}$ be a zero-point of $F^{\prime}(z)$ other than $w_{1}, \cdots, w_{N}$ and $G(-1)$. Then by (3.12), all the roots of $G(z)=s^{*}$ must satisfy $g(z)=1+F\left(s^{*}\right)$. It thus follows from (3.14) that the two equations

$$
\begin{aligned}
& g(z)=z e^{z}=1+F\left(s^{*}\right), \\
& Q\left(s^{*}\right) \exp \left(a^{*} z+b^{*}\right)=1+F\left(s^{*}\right)-c_{m}
\end{aligned}
$$

have infinitely many common roots. By this fact and by the value distribution of $g(z)$, we therefore assert that $a^{*}=0$. Hence

$$
g^{\prime}(z)=\exp \left(b^{*}\right) Q^{\prime}(G(z)) G^{\prime}(z),
$$

so that $g^{\prime}(u)=0$. This is a contradiction. Accordingly, $G^{\prime}(-1)=0$ unless $G(z)=z$. Hereafter we assume that $G^{\prime}(-1)=0$.

Secondly we want to prove that $G(z)$ converges to the value 0 as $z$ tends to infinity along the negative real axis. In order to prove this fact, let us consider the two curves

$$
I=\{G(x): x \geqq-1\}, \quad J=\{G(x): x \leqq-1\},
$$

where $x$ stands for the real variable. Then these curves are simple and smooth, since $G^{\prime}(x) \neq 0$ for real values of $x$ except for $x=-1$. Furthermore by (3.2), the function $F(z)$ maps the curve $I$ to the half straight line $\{t: t \geqq$ $H(-1)\}$ on the real axis, and maps the curve $J$ to the segment $\{t: H(-1) \leqq t<1\}$ on the real axis. Evidently $F^{\prime}(z) \neq 0$ at every point of $I$ and $J$. In particular, $F^{\prime}(G(-1)) \neq 0$. It therefore follows that $J$ coincides with a part of $I$ and $J$ ends at the point $G(0)=0$. This is the desired result. Hence using the value distribution of $g(z)$, we easily see that for an arbitrarily fixed number $t^{*}$ with $0<t^{*}<\pi / 2$,

$$
\lim _{r \rightarrow+\infty} G\left(r e^{i t}\right)=0
$$

uniformly for $|t-\pi| \leqq t^{*}$. Here recall the relation

$$
F^{\prime}(G(z)) G^{\prime}(z)=(1+\exp (g(z))) g^{\prime}(z) .
$$

Then (3.15) implies

$$
\lim _{r \rightarrow+\infty} \frac{G^{\prime}\left(r e^{i t}\right)}{g^{\prime}\left(r e^{2 t}\right)}=1
$$

uniformly for $|t-\pi| \leqq t^{*}<\pi / 2$. Hence by a simple estimation, we also obtain

$$
\lim _{r \rightarrow+\infty} \frac{G\left(r e^{i t}\right)}{g\left(r e^{i t}\right)}=1
$$

uniformly for $|t-\pi| \leqq t^{*}<\pi / 2$. It thus follows that 


$$
\begin{aligned}
& \liminf _{r \rightarrow \infty} \frac{m\left(r, 0, G^{\prime}\right)}{r} \geqq \frac{1}{\pi}, \\
& \liminf _{r \rightarrow \infty} \frac{m(r, 0, G)}{r} \geqq \frac{1}{\pi} .
\end{aligned}
$$

Assume now that $E_{n}$ contains two points $w_{1}$ and $w_{2}$. Since $F^{\prime}(0)=2$, neither $w_{1}$ nor $w_{2}$ is equal to 0 . Then all the roots of $G(z)=w_{\text {, }}$ are also roots of $g(z)=c_{n}(j=1,2)$. Hence

$$
N\left(r, w_{1}, G\right)+N\left(r, w_{2}, G\right) \leqq N\left(r, c_{n}, g\right),
$$

so that the second main theorem yields

$$
(1+o(1)) T(r, G)+N\left(r, 0, G^{\prime}\right) \leqq N\left(r, c_{n}, g\right)
$$

for values of $r$. Therefore

$$
\liminf _{r \rightarrow \infty} \frac{T(r, G)}{r}+\limsup _{r \rightarrow \infty} \frac{N\left(r, 0, G^{\prime}\right)}{r} \leqq \frac{1}{\pi} .
$$

Combining this with (3.16), we thus find $N\left(r, 0, G^{\prime}\right)=o(r)$, so that

$$
\lim _{r \rightarrow \infty} \frac{T(r, G)}{r}=\frac{1}{\pi} \text {. }
$$

Hence (3.16) also gives $N(r, 0, G)=o(r)$. By these facts, we have

$$
\lim _{r \rightarrow \infty} \frac{N(r, w, G)}{r}=\frac{1}{\pi}
$$

for every finite complex number $w$ unless $w=0$. However this clearly contradicts (3.17). Hereby each set $E_{n}$ does not contain more than one point.

Next let us assume that $E_{n}$ is empty. Then all the roots of $g(z)=c_{n}$ must satisfy $G^{\prime}(z)=0$. Hence

so that

$$
N\left(r, c_{n}, g\right) \leqq N\left(r, 0, G^{\prime}\right),
$$

$$
\liminf _{r \rightarrow \infty} \frac{N\left(r, 0, G^{\prime}\right)}{r} \geqq \frac{1}{\pi} \text {. }
$$

Combining this with (3.16), we at once obtain

$$
\liminf _{r \rightarrow \infty} \frac{T(r, G)}{r} \geqq \liminf _{r \rightarrow \infty} \frac{T\left(r, G^{\prime}\right)}{r} \geqq \frac{2}{\pi} .
$$

On the other hand by the second main theorem, it follows that

$$
\begin{aligned}
(1+o(1)) T(r, G)+N\left(r, 0, G^{\prime}\right) & \leqq N(r, a, G)+N(r, b, G) \\
& \leqq N(r, 1+F(a), g)+N(r, 1+F(b), g)+\log ^{+} r,
\end{aligned}
$$

where $a$ and $b$ are two different zeros of $F^{\prime}(z)$. Therefore 


$$
\liminf _{r \rightarrow \infty} \frac{T(r, G)}{r}+\liminf _{r \rightarrow \infty} \frac{N\left(r, 0, G^{\prime}\right)}{r} \leqq \frac{2}{\pi} .
$$

This is impossible by (3.18) and (3.19). Consequently, each set $E_{n}$ consists of only one zero-point of $F^{\prime}(z)$.

The sixth step. Let $w^{*}$ be the point of the set $E_{0}$. Then by the definition (3.13),

$$
F^{\prime}\left(w^{*}\right)=0, \quad F\left(w^{*}\right)=\imath \pi-1 .
$$

Further all the roots of $G(z)=w^{*}$ satisfy $g(z)=\imath \pi$. Conversely, since the set $E_{0}$ consists of only the point $w^{*}$, each root of $g(z)=\imath \pi$ is either a root of $G(z)=w^{*}$ or else a zero-point of $G^{\prime}(z)$. Now with $\left\{a_{n}\right\}$, we denote all the roots of $g(z)=\imath \pi$. These points are distributed on the level line $L(\pi)=\{z$ : $|g(z)|=\pi\}$. Here we may assume that $\operatorname{Re} a_{1}>0, a_{2}=-i \pi$ and $\operatorname{Re} a_{n}<0$ for $n \geqq 3$. Evidently, $g^{\prime}(z) \neq 0$ unless $z=-1$. Hence by using the inverse function of $g(z)$, for each natural number $n$, we can define the simple smooth curve

$$
L_{n}=\left\{z_{n}(t): 0 \leqq t<1\right\}
$$

such that $z_{n}(0)=a_{n}$ and

$$
g\left(z_{n}(t)\right)=\imath \pi(1-t)
$$

for real values of $t$ with $0 \leqq t<1$. All these curves $L_{n}$ are contained in the simply connected domain $\{z:|g(z)|<\pi\}$ except for their initial points. The curve $L_{1}$, which starts from $z_{1}(0)=a_{1}$, must end at the origin. Furthermore except for this $L_{1}$, every curve $L_{n}$ satisfies

$$
\lim _{t \rightarrow 1} \operatorname{Re} z_{n}(t)=-\infty, \quad \lim _{t \rightarrow 1} \arg z_{n}(t)=\pi .
$$

These facts can be verified by an elementary estimation. Hence by (3.15), we can see that

$$
\lim _{t \rightarrow 1} G\left(z_{n}(t)\right)=0
$$

for each curve $L_{n}$. Therefore the function $G(z)$ maps $L_{n}$ to the curve

$$
G\left(L_{n}\right)=\left\{G\left(z_{n}(t)\right): 0 \leqq t<1\right\},
$$

which starts from $G\left(a_{n}\right)$ and converges to the origin. On the other hand by virtue of (3.2) and (3.20), it is clear that

$$
\begin{aligned}
F\left(G\left(z_{n}(t)\right)\right) & =f(i \pi(1-t)) \\
& =i \pi(1-t)-\exp (-i \pi t)
\end{aligned}
$$

for real values of $t$ with $0 \leqq t<1$. Since $f(i \pi(1-t))$ is univalent for $0 \leqq t \leqq 1$ and

$$
F^{\prime}\left(G\left(z_{n}(t)\right)\right) G^{\prime}\left(z_{n}(t)\right) z_{n}^{\prime}(t)=i \pi \exp (-i \pi t)-i \pi,
$$


we can see that every curve $G\left(L_{n}\right)$ is simple and smooth, and that $F^{\prime}(z) \neq 0$ at every point of the curve $G\left(L_{n}\right)$ with the possible exception of the point $G\left(a_{n}\right)$. Here let us note the assumption $F^{\prime}(0)=2$. Then by using the inverse function of $F(z)$ and by (3.21), we can conclude that the curves $G\left(L_{n}\right)$ must coincide with one another. In particular, $G\left(a_{n}\right)=G\left(a_{n+1}\right)$ for every natural number $n$. For a moment we assume that $G\left(a_{n}\right) \neq w^{*}$. Then since the set $E_{0}$ consists of only the point $w^{*}$, all the roots of $g(z)=\imath \pi$ must be zeros of $G^{\prime}(z)$. Accordingly (3.18) and (3.19) hold. We thus have a contradiction. Hence $G\left(a_{n}\right)=w^{*}$ for each natural number $n$. Therefore the functions $G(z)-w^{*}$ and $g(z)-\imath \pi$ have the same zeros. It thus follows that

$$
\frac{g(z)-\imath \pi}{G(z)-w^{*}}=\exp (A z+B)
$$

where $A$ and $B$ are suitable constants. Furthermore by (3.15),

$$
\lim _{r \rightarrow+\infty} \exp \left(A r e^{i t}+B\right)=\frac{2 \pi}{w^{*}}
$$

for real values of $t$ with $\pi / 2<t<3 \pi / 2$. Hence $A=0$, so that (3.22) implies

$$
G(z)=A^{*} g(z)+B^{*},
$$

where $A^{*}$ and $B^{*}$ are constants with $A^{*} \neq 0$. Consequently $G(z)=g(z)$, which is to be proved. Now we have shown that $f(g(z))$ is uniquely factorizable relative to the family of entire functions.

The final step. Let $F^{*}(z)$ and $G^{*}(z)$ be nonconstant meromorphic functions which satisfy

$$
H(z)=f(g(z))=F^{*}\left(G^{*}(z)\right)
$$

for each complex number $z$. If $F^{*}(z)$ is entire and $G^{*}(z)$ has poles, then it is clearly absurd by (3.23).

Assume that $G^{*}(z)$ is entire and $F^{*}(z)$ is not entire. Then by $(3.23), F^{*}(z)$ has exactly one pole $s$ and $G^{*}(z)-s$ never vanishes. Hence

$$
\begin{aligned}
& A(z)=(z-s)^{n} F^{*}(z), \\
& B(z)=\log \left(G^{*}(z)-s\right)
\end{aligned}
$$

are both entire functions with $n \geqq 1$ and $A(s) \neq 0$. It thus follows from (3.23) and (3.24) that

$$
\begin{aligned}
& H(z)=f(g(z))=A^{*}(B(z)), \\
& A^{*}(z)=e^{-n z} A\left(s+e^{z}\right) .
\end{aligned}
$$

Since $n \geqq 1$ and $A(s) \neq 0$, the entire function $A^{*}(z)$ does not reduce to a polynomial. Therefore by the result of the above steps, (3.25) implies either $B(z)$ is linear or else

$$
f(z)=A^{*}\left(B^{\prime}(0) z+B(0)\right) .
$$


Since $A^{*}(z)$ is periodic and $f(z)$ is not periodic, the latter never occurs. Hence $B(z)$ must be linear, so that

$$
H(z)=f(g(z))=A^{*}(a z+b),
$$

where $a$ and $b$ are constants with $a \neq 0$. Evidently

$$
\begin{aligned}
& \lim _{r \rightarrow+\infty} H\left(r e^{i t}\right)=f(0)=1, \\
& \lim _{r \rightarrow+\infty} A^{*}\left(r e^{i t}\right)=\infty
\end{aligned}
$$

for real values of $t$ with $\pi / 2<t<3 \pi / 2$. By this fact and (3.26), the number $a$ is real negative. It therefore follows from (3.25) that

$$
\lim _{x \rightarrow+\infty} A^{*}(a x+b) \exp (n a x+n b)=A(s),
$$

so that

$$
\lim _{x \rightarrow+\infty} H(x) \exp (n a x+n b)=A(s) .
$$

This is a contradiction.

Assume that neither $F^{*}(z)$ nor $G^{*}(z)$ is entire. Then by the relation (3.23), $F^{*}(z)$ reduces to a rational function which has at most two poles and is bounded at a neighborhood of the point at infinity. If $F^{*}(z)$ has two poles $u$ and $v$, then $F^{*}(z)$ can be written in the form

$$
F^{*}(z)=(z-u)^{-m}(z-v)^{-n} P(z),
$$

where $m$ and $n$ are natural numbers, $P(z)$ is a polynomial with

$$
P(u) P(v) \neq 0, \quad \operatorname{deg} P(z) \leqq m+n .
$$

Further with a suitable entire function $S(z)$, we can express $G^{*}(z)$ as

$$
\frac{G^{*}(z)-u}{G^{*}(z)-v}=\exp (S(z)) \text {. }
$$

Combining (3.27) and (3.28), we therefore have

$$
H(z)=(u-v)^{-m-n}\left(1-e^{S(z)}\right)^{m+n} e^{-m S(z)} P\left(G^{*}(z)\right) .
$$

Here let us define the polynomial

$$
Q(z)=\sum_{k=0}^{q} a_{k}(u-v z)^{k}(1-z)^{m+n-k},
$$

where $P(z)=a_{0}+a_{1} z+\cdots+a_{q} z^{q}$ with $a_{q} \neq 0$. Since $0 \leqq q \leqq m+n$ and $P(u) P(v) \neq 0$, the degree of $Q(z)$ must be $m+n$. Furthermore $Q(0) \neq 0$. It thus follows from (3.29) that

$$
\begin{aligned}
& H(z)=f(g(z))=L(S(z)), \\
& L(z)=(u-v)^{-m-n} e^{-m z} Q\left(e^{2}\right) .
\end{aligned}
$$


Since $L(z)$ is a periodic entire function of order one, we finally obtain

$$
f(z)=L\left(a_{*} z+b_{*}\right)
$$

with constants $a_{*}(\neq 0)$ and $b_{*}$. However it is untenable by the periodicity of $L(z)$. From this observation, the function $F^{*}(z)$ has only one pole s. Hence $F^{*}(z)$ is representable as

$$
F^{*}(z)=(z-s)^{-n} P^{*}(z),
$$

where $n$ is a natural number and $P^{*}(z)$ is a polynomial of degree at most $n$ with $P^{*}(s) \neq 0$. Since $G^{*}(z)-s$ has no zeros,

$$
K(z)=\frac{1}{G^{*}(z)-s}
$$

must be entire. Thus we have

$$
\begin{aligned}
H(z) & =F^{*}\left(G^{*}(z)\right)=(K(z))^{n} P^{*}\left(G^{*}(z)\right) \\
& =\sum_{j=0}^{p} b_{j}(1+s K(z))^{\jmath}(K(z))^{n-\jmath},
\end{aligned}
$$

where $P^{*}(z)=b_{0}+b_{1} z+\cdots+b_{p} z^{p}$ with $b_{p} \neq 0$. Setting

we obtain

$$
Q^{*}(z)=\sum_{j=0}^{p} b_{j}(1+s z)^{\jmath} z^{n-\jmath},
$$

$$
H(z)=Q^{*}(K(z)) .
$$

Since $0 \leqq p \leqq n$ and $P^{*}(s) \neq 0, Q^{*}(z)$ is a polynomial of degree $n$. Therefore by the result of the above steps, $Q^{*}(z)$ must be linear. Hence $n=1$, so that $F^{*}(z)$ is a linear transformation. Consequently, the entire function $f(g(z))$ is uniquely factorizable. The proof of Theorem 3 is now complete.

\section{REFERENCES}

[1] Gross, F., Factorization of meromorphic functions, Math. Research Center, Washington D.C., 1972.

[2] Kobayashi, T., Distribution of values of entire functions of lower order less than one, Kōdai Math. Sem. Rep., 28 (1976), 33-37.

[3] Ozawa, M., On uniquely factorizable entire functions, Kōdai Math. Sem. Rep., 28 (1977), 342-360.

[4] Ozawa, M., On uniquely factorizable meromorphic functions, Kodai Math. J., 1 (1978), 339-353.

[5] OzAwa, M., Unique factorizability and permutability of meromorphic functions, Kodai Math. J.,

[6] URABE, H., Uniqueness of the factorization under composition of certain entire functions, J. Math. Kyoto Univ., 18 (1978), 95-120.

[7] YANG, C.C. AND H. URABE, On permutability of certain entire functions, J. London Math. Soc. (2), 14 (1976), 153-159.

Department of Mathematics,

Tokyo Institute of Technology 